\title{
Impacto económico y en el empleo de la Economía Social en España. Un análisis multisectorial
}

\author{
Patricia D. Fuentes Saguar y Alfredo J. Mainar \\ Causapé
}

\section{RESUMEN}

El papel de la economía social en la situación actual resulta muy importante por su capacidad para generar empleo y para dinamizar sectores o colectivos con especiales problemas de desarrollo económico. Por ello, es de gran interés poder medir el alcance real, tanto directo, como indirecto, del impacto de la misma sobre la actividad y el empleo. En este sentido, se propone aquí el uso de modelos multisectoriales, basados en Matrices de Contabilidad Social, para medir dicho impacto total. La combinación de dichos modelos con el uso de la información estadística disponible sobre las empresas de Economía Social en España, permitirá además obtener información sobre la cuantificación de dicho impacto sobre determinados sectores de interés. De esta forma, por otra parte, se podrá también evaluar la capacidad de la economía social para contribuir a la recuperación.

Los resultados muestran que las empresas de Economía Social poseen una capacidad para la creación de empleo superior al resto de empresas en España, especialmente en sectores de servicios. Esto se ve incrementado por la alta capacidad de multiplicar los impactos y la importante ratio de empleo de estos sectores (básicamente de Servicios), ampliado en el caso de la Economía Social.

PALABRAS CLAVE: Economía Social, Empleo, Modelos multisectoriales, Matriz de Contabilidad Social.

CLAVES ECONLIT: E00, E20, P00.

Cómo citar este artículo: FUENTES, P. \& MAINAR, A. (2015): "Impacto económico y en el empleo de la Economía Social en España. Un análisis multisectorial", CIRIEC-España, Revista de Economía Pública, Social y Cooperativa, 83, 63-81.

Correspondencia: Dra. Patricia D. Fuentes Saguar, Departamento de Economía, Universidad Pablo de Olavide, España, e-mail: pfuesag@upo.es; Dr. Alfredo J. Mainar Causapé, Departamento de Economía Aplicada III, Universidad de Sevilla, España, e-mail: amainar@us.es. 


\title{
Impact économique et sur l'emploi de l'économie sociale en Espagne. Une analyse multisectorielle
}

RÉSUMÉ : Le rôle de l'économie sociale dans la situation actuelle est très important du fait de sa capacité à générer de l'emploi et à dynamiser les secteurs ou les collectifs avec des problèmes particuliers de développement économique. Il est donc très intéressant de pouvoir mesurer la portée réelle, directe et indirecte, de l'impact de l'économie sociale sur l'activité et l'emploi. Dans ce sens, cet article propose d'utiliser des modèles multisectoriels basés sur des matrices de comptabilité sociale afin de mesurer cet impact total. L'association de ces modèles avec l'utilisation des informations statistiques disponibles sur les entreprises de l'économie sociale en Espagne permettra également d'obtenir des informations sur la quantification de cet impact sur des secteurs d'intérêt déterminés. De cette manière, il sera également possible d'évaluer la capacité de l'économie sociale à contribuer au redressement.

Les résultats montrent que les entreprises de l'économie sociale possèdent une capacité de création d'emploi supérieure à celle des autres entreprises espagnoles. Particulièrement dans le secteur des services. Ce phénomène est renforcé par la haute capacité de multiplication des impacts et par l'important ratio d'emploi de ces secteurs (principalement de services), plus important encore dans le cas de l'économie sociale.

MOTS CLÉ : Économie sociale, emploi, modèles multisectoriels, matrice de comptabilité sociale.

\section{The economic and employment impact of the Social Economy in Spain - A multi-sector analysis}

\begin{abstract}
In the current situation, the social economy has a very important role to play because of its capacity to create employment and to energise sectors or collectives with particular economic development problems. For this reason, the ability to measure the real scope - both direct and indirect - of its impact on economic activity and employment is a matter of great interest. This paper proposes the use of multi-sector models, based on social accounting matrices, to measure the total impact of the social economy. Combining these models with the available statistical data on social economy enterprises in Spain will also make it possible to obtain information to quantify this impact in particular sectors of interest. Additionally, in this way the capacity of the social economy to contribute to economic recovery can also be assessed.

The results show that social economy enterprises have a greater capacity for job creation than other companies in Spain, particularly in service sectors. This is augmented by the considerable ability of these sectors (essentially services) to multiply impacts and by their high employment ratio, which is still greater in the case of the social economy.
\end{abstract}

KEY WORDS: Social economy, employment, multi-sector models, social accounting matrix. 


\section{1.- Introducción. La Economía Social: su importancia y la necesidad del análisis de su impacto}

La Economía Social hace referencia al conjunto de organizaciones de productores, consumidores, trabajadores y agentes económicos en general que operan bajo los principios de participación democrática en las decisiones, autonomía de la gestión y la primacía del ser humano sobre el capital. Las prácticas de estas organizaciones se circunscriben en una nueva racionalidad productiva, donde la solidaridad es el sostén del funcionamiento de las iniciativas. Estos emprendimientos buscan la articulación con entidades públicas o privadas que les permitan llevar a cabo una verdadera participación democrática en la vida económica y política de una sociedad. En ellas encuentran refugio categorías sociales puestas al margen de los sistemas de empleo y distribución de la riqueza convencionales, dependientes del mercado y del Estado.

La Economía Social incluye también a las instituciones sin fines de lucro, que no siendo de mercado privado, tampoco están controlados por el Sector Público, produciendo servicios no destinados a la venta para ciertos grupos de hogares. Sus recursos provendrían, básicamente, de contribuciones voluntarias efectuadas por los hogares en su calidad de consumidores, de pagos de las Administraciones Públicas y de algunas rentas de la propiedad (Chaves y Monzón, 2000).

En los últimos años, la literatura especializada ha tratado la problemática de la conceptualización y definición de la Economía Social (EOI, 2013; Juliá y Server, 2003). Así, se coincide en señalar la co-existencia a nivel internacional no sólo de diversas terminologías para definir el concepto de Economía Social sino también diferencias en las entidades que la conforman (EOI, 2013). En Alemania se usa la expresión "economía de interés general", que incluye a las empresas públicas, sindicatos y asociaciones. En Estados Unidos las "non-profit organisations" recogen a aquellas entidades que no reparten los beneficios entre sus asociados y asociadas, mientras que en Francia y Bélgica bajo el término "Economía Social" se definen las empresas cooperativistas, mutualistas y asociativas.

Como integrantes de la economía social es posible incluir a las cooperativas, las empresas mutuales, las fundaciones, las organizaciones civiles, las denominadas cooperadoras y las fábricas recuperadas que funcionan como cooperativas en su gran mayoría; pero también a las organizaciones de desocupados que organizan comedores, huertas u otros emprendimientos. En los últimos años, esta línea de acción y pensamiento se ha potenciado con el desarrollo de iniciativas vinculadas a la gestión ética de las empresas y las distintas vertientes de la responsabilidad social empresarial.

Las instituciones pertenecientes a la Economía Social tienen cierto grado de propiedad privada (aunque el Estado puede participar en su propiedad) y no tienen el lucro como único objetivo, sino que tienen un fin social (pueden tener el objetivo de lucro, pero este no es el único). Los límites trazados 
no siempre están perfectamente definidos, pudiendo coexistir en una misma iniciativa empresarial participación, tanto del estado, como del sector privado, con objetivos comerciales y sociales simultáneos.

Entre las principales características de estas iniciativas destacan: la democracia participativa y la autogestión, la práctica de la solidaridad (prioridad para trabajadores en vías de perder el empleo y para los que tienen menos posibilidades de encontrar trabajo debido a la edad, la falta de calificación, discriminación de raza o de género, etc.), el desarrollo local y la sustentabilidad (la economía social se fundamenta en los principios de desarrollo económico respetando el medio ambiente y el compromiso con la cohesión social, siendo pionera en las prácticas de responsabilidad social). En este sentido, es claro su papel cada vez más importante en las políticas de desarrollo en todos los ámbitos (Barrera, 2008).

La importancia del análisis del impacto de la Economía Social en la economía española viene justificada por varios de los aspectos que definen a este sector en su papel dentro del tejido productivo español (CEPES, 2014). Así, se considera que la economía social es un mecanismo generador de empleo de calidad, por cuanto prioriza el bienestar del factor trabajo procurando unir a su productividad una adecuada caracterización de prestaciones de calidad de vida. Actúa, además, con más fuerza allí donde la oferta de empleo decrece.

Por otra parte, este sector reúne y es enriquecedor de empresas, promotor de proyectos de desarrollo empresarial y potenciador de actividades económicas, también en zonas geográficas en las que no existe alternativa, recuperando y promoviendo las iniciativas locales de empleo y riqueza. Es, por lo tanto, la manifestación socio-económica de los que se está reconociendo como "tercer sector", incorporando desde su acción a los diversos niveles sociales para su integración en el llamado "Estado del Bienestar".

Estos factores justifican la necesidad de realizar un estudio que permita cuantificar, de la forma más rigurosa posible, la importancia económica que, de modo cualitativo, ya se intuye que tiene en la recuperación y el crecimiento económico. Una de las formas es la propuesta en este trabajo, en el que se van a utilizar modelos multisectoriales para plasmar el impacto de esta forma de economía sobre la producción y el empleo, cuantificando así su reconocida importancia social.

\section{2.- Metodología. Modelos Input-Output y Matrices de Contabilidad Social}

Los orígenes del análisis Input-Output y de las Matrices de Contabilidad Social (MCS o SAM, acrónimo de su expresión inglesa, Social Accounting Matrix) se remontan a la obra de Quesnay (1758) y su conocida tableau économique. Sin embargo, el verdadero inicio y el desarrollo de estas herramientas de análisis multisectorial se produjo en la primera mitad del siglo XX, con las obras de Leontief 
(1941), que establece la base de las tablas Input-Output y de los modelos lineales y aplicaciones relacionadas, y Kuznets (1937), que delimita los parámetros básicos de los sistemas de cuentas nacionales. La capacidad de descripción, explicación y análisis de la economía que permite el modelo tradicional Input-Output, le ha convertido en una herramienta clave en el estudio económico, mientras que los sistemas de contabilidad nacional se han desarrollado y generalizado para la mayoría de las economías, formando un conjunto de puntos de referencia básicos de la situación económica. Sin embargo, la poderosa herramienta de análisis multisectorial que representa el modelo Input-Output es limitada en el intento de reflejar el pleno funcionamiento del sistema económico, al no incorporar todas las transacciones económicas que se producen en un sistema, ya que no plantea cómo los llamados sectores institucionales (hogares, gobiernos, corporaciones, etc.) llevan a cabo las transacciones y la reasignación de recursos e ingresos. No está recogida, por ejemplo, la distribución del ingreso de los factores de producción entre aquellos que los ofrecen (sectores institucionales), que son precisamente los que llevan a cabo la demanda de bienes y servicios que forman la reasignación de los recursos. Así, los modelos Input-Output no contemplan suficientemente el flujo circular de la renta ni los procesos de retroalimentación subsecuentes de ingresos, obviando su influencia en el comportamiento de los agentes y la composición de la demanda.

Dada esta limitación, las Matrices de Contabilidad Social se pueden definir (de forma simplificada) como una extensión de las tradicionales tablas Input-Output, introduciendo una estructura desglosada de los gastos e ingresos, así como explicitando las relaciones entre los sectores institucionales, utilizando información de los sistemas de contabilidad nacional. De esta manera, se logra el objetivo de cerrar el flujo económico y obtener una descripción completa y real de la economía de un país o región. Así, una SAM es un marco consistente de datos que recoge los datos de la renta nacional, de los niveles de producción, de las relaciones intersectoriales y de los flujos monetarios entre las instituciones. Por lo tanto, una SAM es una representación matricial de las transacciones de un sistema socio-económico, en un marco amplio, flexible y desglosado, reflejando el proceso de generación de ingresos de las actividades, de la producción y la distribución y redistribución de la renta entre los grupos institucionales. Las diversas interdependencias en el sistema económico se toman en su conjunto mediante el registro de las transacciones y las transferencias entre los agentes del sistema de efectivos e imputados. El punto clave que distingue el uso de una SAM frente a los sistemas contables alternativos es la importancia de los factores, de los hogares y de las dimensiones institucionales, recopilados de manera integral (Pyatt \& Round, 1985; Pyatt y Thorbecke, 1976).

Puede ser considerado como el génesis de las Matrices de Contabilidad Social el trabajo pionero sobre las cuentas sociales de Stone (1947), en el que ya se muestra un sistema integrado de cuentas nacionales, siendo la base de la mayoría de los convenios que han sido seguidos por organismos económicos y estadísticos en el desarrollo de esta herramienta. En cualquier caso, es a partir de la obra de Pyatt y Thorbecke (1976) cuando el concepto de lo que es una SAM fue formalizado, lo que permite su uso como un marco formal para el análisis económico y la planificación. Posteriormente, también es crucial a este respecto el estudio de Pyatt y Round (1985). 
Una SAM proporciona un marco importante para el análisis de los principales temas socioeconómicos como el empleo, la pobreza, el crecimiento y la distribución del ingreso, etc. Mediante la integración de los datos de encuestas de hogares en las cuentas nacionales, la SAM captura las transacciones a nivel macro de un sistema económico y las relaciones de nivel micro implícitas en las transferencias entre todos los agentes económicos en la economía (Pyatt y Round, 1985; Reinert y Roland-Holst, 1997). Por otra parte, una SAM incorpora dimensiones de distribución del ingreso mediante la desagregación de los hogares por sus características socio-económicas.

Más allá de la elaboración y la representación estadística detallada del flujo circular de la renta, un SAM debe proporcionar información sobre cómo los diferentes agentes de una economía interactúan con el resto del sistema económico. Por lo tanto, la compilación de una SAM requiere una estimación detallada y uso de los conjuntos de datos que no figuren en la práctica de la contabilidad nacional estándar, lo que ya es una contribución importante en sí misma. Dado que los datos necesarios para construir una MCS útil no están disponibles en una sola fuente, la información de distinta procedencia se debe compilar y hacer coherente. Este proceso es en sí mismo valioso, ya que muestra inconsistencias entre las fuentes estadísticas y las áreas donde la fiabilidad de los datos es más débil, aportando soluciones. Además, y esto es fundamental en su desarrollo, representa un marco analítico necesario para la modelización, siendo la base de datos para los modelos directos de multiplicadores lineales y para la calibración de los Modelos de Equilibrio General Aplicado (MEGAs). Además, el interés de las SAMs aumenta por las posibilidades de comparación, temporal y espacial, entre los sistemas económicos que permiten estas bases de datos, así como por su posible aplicación en todo tipo de cuestiones relacionadas con la estructura socio-económica del territorio: el medio ambiente, el empleo, la productividad, la pobreza, el desarrollo, el cambio tecnológico, etc.

\subsection{Fundamentos y estructura básica de una SAM}

Una SAM representa la economía real de un territorio. Es una matriz cuadrada en la que cada cuenta está representada por una fila y columna. Cada celda muestra el pago de la cuenta de su columna a la cuenta de su fila. Así, los ingresos de una cuenta se muestran a lo largo de su fila y sus gastos a lo largo de su columna. Por el principio de contabilidad de doble entrada, en una SAM el total de los ingresos debe ser igual al total de los pagos totales de cada cuenta, por lo que los ingresos totales (total fila) son iguales a los gastos totales (total de la columna).

Por lo general, un SAM cuenta con seis grupos básicos de cuentas: Actividades / Productos, Factores de producción, Instituciones privadas, Gobierno, Cuentas de capital y Resto del Mundo. Las dimensiones de la matriz son determinadas por el nivel de desagregación de estos seis grupos básicos. El Cuadro 1 muestra la estructura básica de una SAM, con las instituciones privadas desglosadas en Hogares y Empresas y tomando por separado Actividades y Productos. 
Esta estructura simplificada es suficiente para mostrar la compleja obra necesaria en la recopilación de datos para una SAM. Sólo en unos pocos países en desarrollo las cuentas nacionales se calculan sobre la base de los ingresos o los métodos de gasto. Además, hasta periodos recientes no era habitual encontrar balances de productos (por ejemplo, las tablas Input-Output) o integrar los datos sobre los ingresos y gastos de los hogares, empresas o gobiernos.

Por lo general, hay dos pasos principales en la construcción de una SAM. En primer lugar, es necesario recopilar información de diversas fuentes para formar una Macro-SAM con información agregada de las Cuentas Nacionales y otras bases de datos. Esta Macro-SAM es luego desagregada, en una segunda etapa, a través de las actividades, productos, los diferentes tipos de factores (trabajo, capital, etc.), grupos de hogares, impuestos, otros sectores institucionales, el resto del mundo desagregado (por regiones o grupos de países) o no, etc. Esta tarea concluye con una detallada Micro-SAM que, por lo general, es inconsistente (existen desigualdades entre los ingresos y pagos), debido a las diferentes fuentes utilizadas. Para finalizar el procedimiento, es necesario conciliar los ingresos y pagos (es decir, "el equilibrio de la SAM") a partir de datos estadísticos adicionales o con la ayuda de métodos econométricos como el RAS o el Cross-Entropy Method (McDougall, 1999; Robinson et al., 2001).

Cuando la construcción de una nueva SAM para un período de tiempo o para una región en particular no es viable por falta de presupuesto o tiempo, se utilizan métodos estadísticos para actualizar una SAM anterior y reflejar en ella los nuevos datos macroeconómicos (por ejemplo, el PIB, el ahorro, los saldos de la Balanza de Pagos ). Este enfoque asume que las características estructurales de la economía no han cambiado significativamente con el tiempo o en el espacio geográfico, un supuesto importante ya que uno de los puntos fuertes de una SAM es su captación de la tecnología de producción y los vínculos entre los diferentes sectores / instituciones en la economía. Por ello, no es apropiado durante un período de transformación, pero puede ser muy útil para las economías desarrolladas (o regiones similares) y por períodos no tan lejanos en el tiempo de una matriz original.

Como se mencionó, la construcción de una SAM requiere una gran cantidad de información estadística, pero en su preparación, una parte fundamental es la estimación de las interrelaciones entre los sectores productivos. Estas interrelaciones pueden ser tomadas directamente de la matriz de consumos intermedios de las tablas Input-Output tradicionales, simétricas, suponiendo que las ramas productivas son homogéneas (cada actividad sólo produce un tipo de bien o producto o servicio), o bien hacer la distinción entre las actividades productivas propiamente dichas y los bienes y servicios que producen, como se propone en la mayoría de la sistemas de cuentas actuales (por ejemplo, el Sistema Europeo de Cuentas Nacionales y Regionales, de la ESA, 2010 (European Commission, 2013)), utilizando en este caso las tablas de Uso y Destino (Supply and Use Input-Output Tables, SUIOT), con tablas no cuadradas. En cualquier caso, la dificultad del cálculo de cualquiera de estas matrices es la razón por la que la mayoría de la SAMs (si no son una actualización) se construyen para el mismo período para el cual se dispone de los correspondientes marcos Input-Output. 


\begin{tabular}{|c|c|c|c|c|c|c|c|c|c|c|}
\hline & $\underline{\underline{v}}$ & 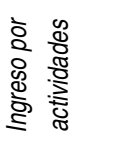 & 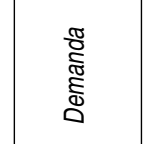 & 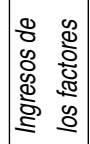 & 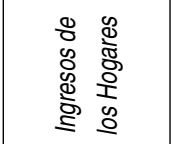 & 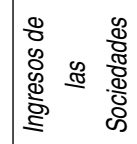 & 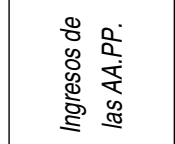 & $\begin{array}{l}0 \\
\frac{2}{4}\end{array}$ & 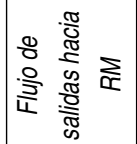 & \\
\hline & 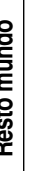 & & 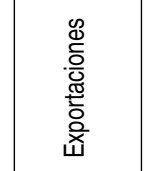 & 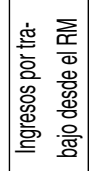 & 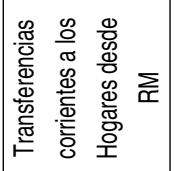 & 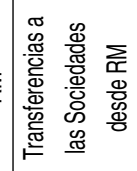 & 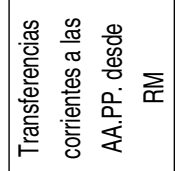 & 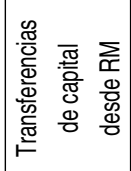 & & 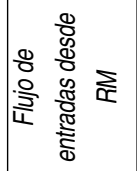 \\
\hline & 这 & & 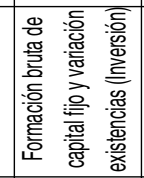 & & & & & 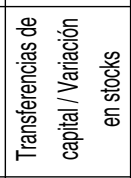 & 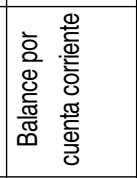 & 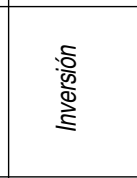 \\
\hline & 竞 & & 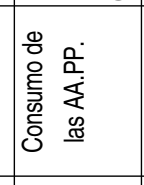 & & 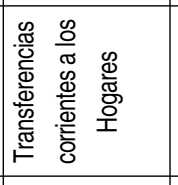 & 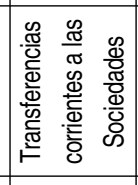 & & 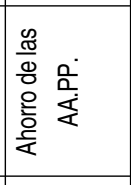 & 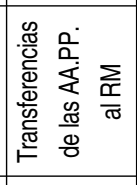 & 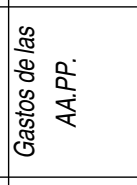 \\
\hline & 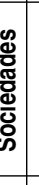 & & & & 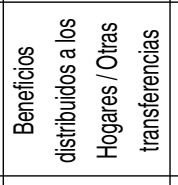 & & 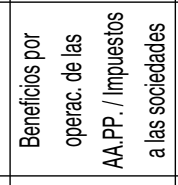 & 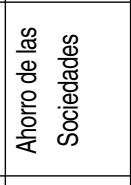 & 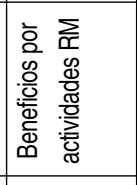 & 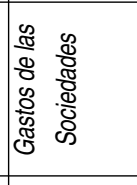 \\
\hline & 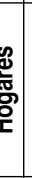 & 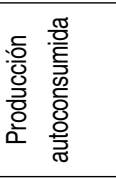 & 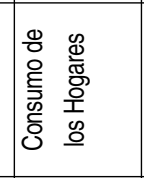 & & & & 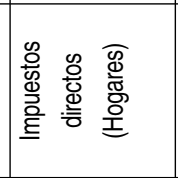 & 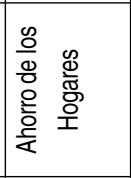 & 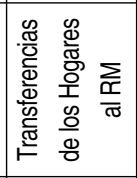 & 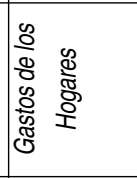 \\
\hline & 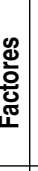 & & & & 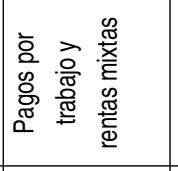 & 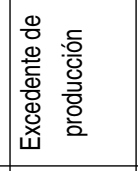 & 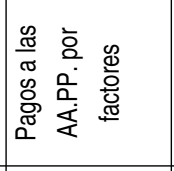 & & 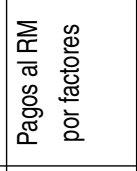 & 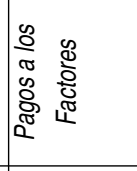 \\
\hline & : & 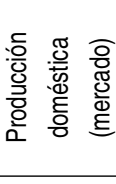 & 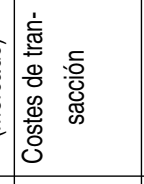 & & & & 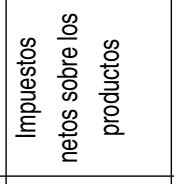 & & 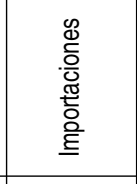 & $\begin{array}{l}\frac{\pi}{50} \\
\frac{\pi}{0}\end{array}$ \\
\hline & 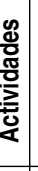 & & 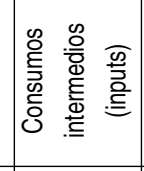 & 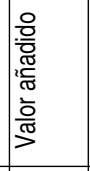 & & & & & & 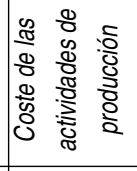 \\
\hline & & 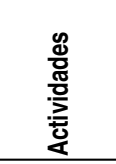 & 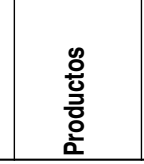 & 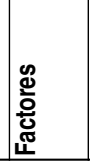 & 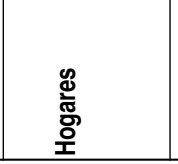 & 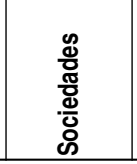 & $\frac{0}{2}$ & 它: & 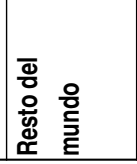 & 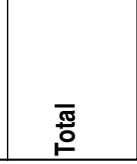 \\
\hline
\end{tabular}




\subsection{Modelos lineales SAM}

Con el desarrollo de una SAM se puede realizar la extensión correspondiente del análisis de multiplicadores empleado habitualmente con las tablas Input-Output. El modelo lineal simple basado en la llamada inversa de Leontief ofrece multiplicadores que indican el impacto en los niveles de producción de cada actividad productiva de los cambios en la demanda final. Sin embargo, los efectos causados en las variaciones en la distribución de los ingresos de los hogares y el sector público, como resultado de los cambios experimentados por la actividad económica o en los ingresos, no son considerados por estos multiplicadores de Leontief. Esta limitación se supera con el uso de las SAM, cuyos multiplicadores incluyen los efectos inducidos por la interdependencia que surge de la consideración del flujo total de la economía. Permite, además, el análisis de varias cuestiones pertinentes con características sociales, como las emisiones de $\mathrm{CO}_{2}$, la pobreza y la desigualdad, la generación de empleo, cuestiones de género, etc.

Como se ha señalado anteriormente, estas matrices pueden usarse como simple elemento descriptor del sistema de transacciones y flujos en una economía, o ser utilizado como base de datos de modelos más o menos complejos. Una de las aplicaciones más habitual consiste en el cálculo, de forma análoga al modelo Input-Output, de los llamados multiplicadores contables, que recogen el efecto total sobre cada una de las partidas consideradas endógenas en la SAM de un impacto unitario adicional en el conjunto de las variables exógenas. Este será el modelo base utilizado en este trabajo. En este sentido, el punto básico de referencia en este trabajo será la Matriz de Contabilidad Social de España del año 2008 (SAMESP-08), elaborada por los autores (Fuentes y Mainar, 2014). Disponer de una base de datos del tipo de la SAMESP-08, con gran cantidad de información desagregada y con un elevado grado de fiabilidad, permite que los análisis basados en la misma sean suficientemente sólidos como para servir de soporte en un estudio riguroso de la situación social y económica del país, así como para la toma de decisiones de política económica en el corto, medio e incluso en el largo plazo.

La herramienta analítica básica para lograr el objetivo de realizar un análisis en profundidad de la economía social en España a partir de la SAMESP-08, será el desarrollo y estimación de multiplicadores lineales y de los coeficientes de arrastre e impulso, y la estimación de impactos en el empleo.

La formulación de los modelos lineales de los que se obtienen los multiplicadores parte de la expresión general siguiente:

$$
y_{n}=A_{n} y_{n}+x=(I-A)^{-1} x=M_{a} x
$$

donde $y_{n}$ es el vector columna de rentas de las cuentas endógenas, $A_{n}$ sería la matriz de propensiones medias al gasto de las diferentes cuentas endógenas, cuyos componentes $a_{i j}$ representarían el gasto realizado en la cuenta i por cada unidad monetaria de gasto 0 empleo total de $j$, siendo $x$ el vector columna que contabiliza el total de los flujos de renta que las cuentas endógenas reciben de las cuentas exógenas.

1.- Una descripción más detallada de esta metodología, así como sendas aplicaciones de la misma, pueden verse en Cardenete et al. (2015) o en Fernández y González (2004). 
La matriz $M_{a}$ es la matriz de multiplicadores contables, cuyos componentes $\left(m_{\text {aij }}\right)$ reflejan el impacto que una unidad exógena de renta sobre una cuenta endógena $j$, genera finalmente, tras recorrer el circuito del flujo circular de la renta sucesivas veces, sobre la renta de la cuenta endógena $i$. La suma de las columnas de la matriz de multiplicadores contables indicaría el efecto total de un shock exógeno recibido por una cuenta endógena sobre el resto de la actividad económica. Por ello, una columna de esta matriz cuya suma fuera un valor muy alto está indicando una cuenta que tiene una gran influencia sobre el resto de la economía cuando recibe un shock exógeno, que puede ser provocado por una política económica determinada, o por otro acontecimiento externo.

Una vez obtenidos estos multiplicadores, pueden determinarse algunos de los coeficientes más habitualmente utilizados en el análisis Input-Output clásico, como aquellos que recogen los efectos absorción y difusión, los definidos por Rasmussen (1956) y los que hacen referencia a la homogeneidad en la transmisión de dichos efectos. Los coeficientes difusión 0 arrastre se obtienen mediante la suma de los elementos de cada columna de la matriz de multiplicadores, es decir, $\mathrm{M}_{\mathrm{j}}=\sum_{\mathrm{i}=1}^{\mathrm{n}} \mathrm{m}_{\mathrm{ij}}$ Esta expresión proporciona información sobre aquellas cuentas que presentan mayores efecios ae expansión sobre la renta de la economía en su conjunto, puesto que su valor indica en cuánto se incrementa la renta del total de las cuentas endógenas cuando se produce un aumento unitario exógeno de renta en la cuenta j.

Los coeficientes de absorción se derivan sumando los elementos de cada fila de la matriz de contabilidad social, $\mathrm{M}_{\mathrm{i}}=\sum_{\mathrm{i}=1}^{\mathrm{n}} \mathrm{m}_{\mathrm{ij}}$, y muestran aquellas cuentas que absorben una mayor parte del crecimiento que se produce en la renta. Su valor indica en cuánto aumenta la renta de la cuenta i cuando se produce una inyección unitaria exógena de renta en el sistema económico.

Los denominados coeficientes de Rasmussen normalizan $\mathrm{M}_{\mathrm{i}}$ y $\mathrm{M}_{\mathrm{i}}$ al compararlos con el promedio global y, por lo tanto, proporcionan una medida relativa de la "fuerza" de los efectos absorción y difusión. Así pues, se pueden definir

$$
\mathrm{U}_{\mathrm{j}}=\frac{\frac{1}{\mathrm{n}} \sum_{\mathrm{i}=1}^{\mathrm{n}} \mathrm{m}_{\mathrm{ij}}}{\frac{1}{\mathrm{n}^{2}} \sum_{\mathrm{j}=1}^{\mathrm{n}} \sum_{\mathrm{i}=1}^{\mathrm{n}} \mathrm{m}_{\mathrm{ij}}}=\frac{\mathrm{M}_{\mathrm{j}}}{\frac{1}{\mathrm{n}} \sum_{\mathrm{j}=1}^{\mathrm{n}} \sum_{\mathrm{i}=1}^{\mathrm{n}} \mathrm{m}_{\mathrm{ij}}}
$$

que recogen la fuerza difusora, en términos relativos, de cada cuenta considerada. Estos serían los llamados Backward Linkages (BL). En este mismo sentido puede expresarse:

$$
U_{i}=\frac{\frac{1}{n} \sum_{j=1}^{n} m_{i j}}{\frac{1}{n^{2}} \sum_{j=1}^{n} \sum_{i=1}^{n} m_{i j}}=\frac{M_{i .}}{\frac{1}{n} \sum_{j=1}^{n} \sum_{i=1}^{n} m_{i j}}
$$

como el coeficiente que mide la importancia, en términos relativos, de las cuentas que absorben un incremento en la renta. A estos coeficientes se les denomina Forward Linkages (FL). 


\section{3.- Datos}

\subsection{La Matriz de Contabilidad de España 2008 (SAMESP-08)}

En este trabajo se utiliza una SAM para el año 2008 de la economía española, la SAMESP-08, elaborada con anterioridad por los autores (Fuentes y Mainar, 2014). El motivo de elección de este periodo es la disponibilidad, en el momento de comienzo de su elaboración, de un Marco Input-Output sólo hasta el año 2008. Puesto que la última tabla simétrica Input-Output de la economía española (tabla base de las SAM con la estructura de industrias homogéneas, que es la aquí utilizada) corresponde al año 2005, para su construcción se utilizaron las Tablas de Origen y Destino de 2008, obteniendo una matriz simétrica mediante los correspondientes algoritmos (Eurostat, 2008), usando la metodología de industria por industria y con la hipótesis de estructura fija de venta de producto. De esta forma, la SAMESP-08 consta de 84 cuentas:

- 73 Actividades productivas / Productos.

- 2 Factores de producción (Capital y Trabajo)

- 2 Sectores institucionales privados (Hogares y Sociedades).

- 3 cuentas de impuestos netos de subvenciones (sobre la producción, sobre los productos y sobre el ingreso).

- Cuenta del Sector Público / Administraciones Públicas (AA.PP.)

- Cuenta combinada de capital: Ahorro / Inversión.

- 2 cuentas del Sector Exterior: (Unión Europea y Resto del Mundo)

Los sectores productivos se agregan posteriormente para facilitar el análisis de los datos y se toman como variables exógenas, en el presente ejercicio, las cuentas de AA.PP. y Sector Exterior.

\subsection{Delimitación del sector Economía Social}

Las fórmulas jurídicas (tipos de empresas) fundamentales que componen la Economía Social son siete: cooperativas, sociedad laborales, autónomos, empresas de inserción, mutualidades, asociaciones y fundaciones y centros especiales de empleo. De estas, las cuatro primeras son las principalmente utilizadas, existiendo dentro de ellas numerosos tipos y subtipos jurídico-económicos.

Las cooperativas son sociedades participativas que aúnan a personas físicas o jurídicas con intereses o necesidades socioeconómicos comunes, para cuya satisfacción y en interés de la comunidad realizan cualquier actividad empresarial. Las sociedad laborales son aquellas sociedades anónimas 0 
de responsabilidad limitada de naturaleza mercantil, en las que la mayoría del capital social es propiedad de las personas que trabajan en ellas, prestando servicios retribuidos en forma personal y directa y cuya relación laboral es por tiempo indefinido.

La persona trabajadora por cuenta propia o autónoma es aquella que realiza de forma habitual, personal y directa una actividad económica a título lucrativo, sin sujeción por ella a contrato de trabajo aunque utilice el servicio remunerado de otras personas. Finalmente, las empresas de inserción ${ }^{2}$ surgen como un instrumento para luchar contra la pobreza y la exclusión social.

Para el presente análisis se han tomado los datos de empresas y trabajadores afiliados a la Seguridad Social en Cooperativas y Sociedades Laborales, puesto que son los que pone a disposición general de los usuarios el Ministerio de Empleo y Seguridad Social en sus Anuarios Estadísticos (MEYSS, varios años) y están desagregados por sectores productivos. Aunque no representan a la totalidad de la Economía Social en España, sí que son la parte más significativa de la misma, cuando menos cuantitativamente (CEPES, 2014). A modo ilustrativo, el Gráfico 1 muestra la distribución por grandes sectores de los trabajadores de esta parte de la Economía Social:

\section{Gráfico 1. Distribución en España, por grandes sectores de actividad, de los trabajadores de Cooperativas y Sociedades Laborales. Media 2011-2013}

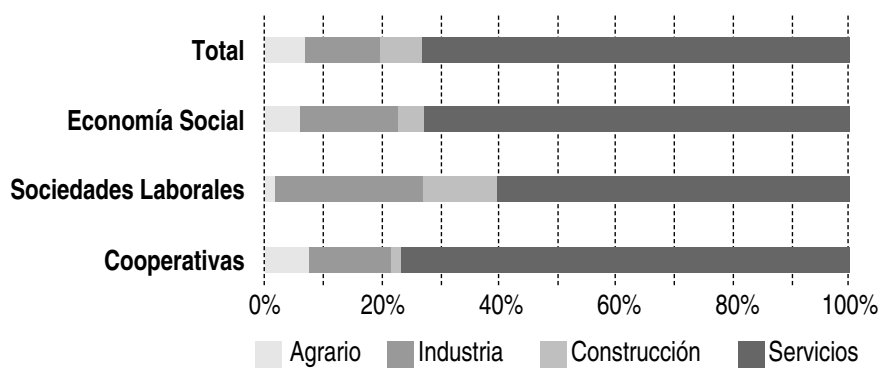

FUENTE: Elaboración propia a partir de datos del Anuario estadístico del MEYSS (varios años).

Estos datos de empleo, convenientemente distribuidos entre las categorías que los delimitan y transformados en coeficientes de empleo por unidad (monetaria) de producto, se han combinado con los multiplicadores obtenidos previamente de la SAMESP-08, permitiendo evaluar el impacto que tiene en el empleo la Economía Social.

2.- Son iniciativas que combinan la lógica empresarial con metodologías de inserción laboral en la misma empresa, en una ajena o en proyectos de autoempleo. No están al margen de los procesos convencionales de la economía, ya que producen bienes y servicios, mejoran el entorno, potencian los servicios a las personas y favorecen la calidad de vida, siendo rentables y competitivas. Además de valorar su rentabilidad económica es muy importante destacar la rentabilidad en los aspectos sociales, ya que los beneficiarios dejan de ser personas pasivas y dependientes y aportan a la sociedad todo aquello que ésta les había negado. Sus destinatarios son personas en situación de exclusión que estén inactivas o en paro y que tengan dificultades importantes para acceder a un empleo. 


\section{4.- Resultados}

El análisis planteado para medir cuantitativamente el impacto de la Economía Social en España se ha concretado en una comparación del mismo frente a la economía española en su conjunto, siempre en términos de empleo. Para ello, se han tomado de forma conjunta los datos de trabajadores afiliados y de empresas de cooperativas y sociedades laborales como representativos de la Economía Social, y se ha calculado, para cada uno de los sectores disponibles en la desagregación (básicamente, sectores de actividad de la CNAE 2009), la ratio entre trabajadores y empresas. Dicha ratio se pone en comparación con la media de la economía española, en ambos casos para el periodo 2011-2013.

\section{Tabla 1. Cociente de las ratios de empleo (afiliados a la Seguridad Social) por empresa entre Economía Social y Total de la Economía. España, promedio años 2011-2013}

\begin{tabular}{|l|c|}
\hline Agricultura, ganadería, silvicultura y pesca & 2,65 \\
Industrias extractivas & 0,30 \\
Industria manufacturera & 0,89 \\
Suministro de energía eléctrica, gas, vapor y aire acondicionado / Suministro de agua, saneamiento, gestión residuos & 0,23 \\
Construcción & 1,17 \\
Comercio al por mayor y por menor; reparación vehículos motor & 3,21 \\
Transporte y almacenamiento & 1,58 \\
Hostelería & 1,48 \\
Información y comunicaciones & 0,51 \\
Actividades financieras y de seguros & 10,34 \\
Actividades inmobiliarias & 1,04 \\
Actividades profesionales, científicas y técnicas / Actividades administrativas y servicios auxiliares & 1,32 \\
Administración Pública y Defensa; Seguridad Social obligatoria & 0,06 \\
Educación & 1,99 \\
Actividades sanitarias y de servicios sociales & 1,81 \\
Actividades artísticas, recreativas y de entretenimiento & 1,81 \\
Otros servicios & 2,14 \\
Activ. hogares empleadores personal doméstico, productores bienes y serv. & 0,28 \\
\hline
\end{tabular}

FUENTE: Elaboración propia. 
Simultáneamente, se ha agregado la SAMESP-08 en los mismos sectores de actividad que los disponibles para dichos datos de afiliación y se han obtenido los multiplicadores lineales del tipo Leontief para estos sectores. El Gráfico 2 muestra dichos valores en comparación con la media, de forma que se aprecian cuales son aquellos con mayor capacidad de impacto en la economía española. Así, destacan claramente los sectores de servicios, especialmente la Educación y el Comercio, mientras que todavía existen valores significativos para la Construcción por estar referida la base de datos fundamental (la SAMESP-08) justo al periodo de inicio de la crisis, con un peso todavía relevante del sector. En cualquier caso, esto es igualmente válido para el análisis por cuanto dicha importancia cuantitativa refleja el tirón del sector en la economía, tanto positiva, como negativamente, mostrando el porqué de su responsabilidad en el agravamiento de la crisis en España.

\section{Gráfico 2. Valores de los multiplicadores lineales sectoriales en comparación con la media. España, 2008}

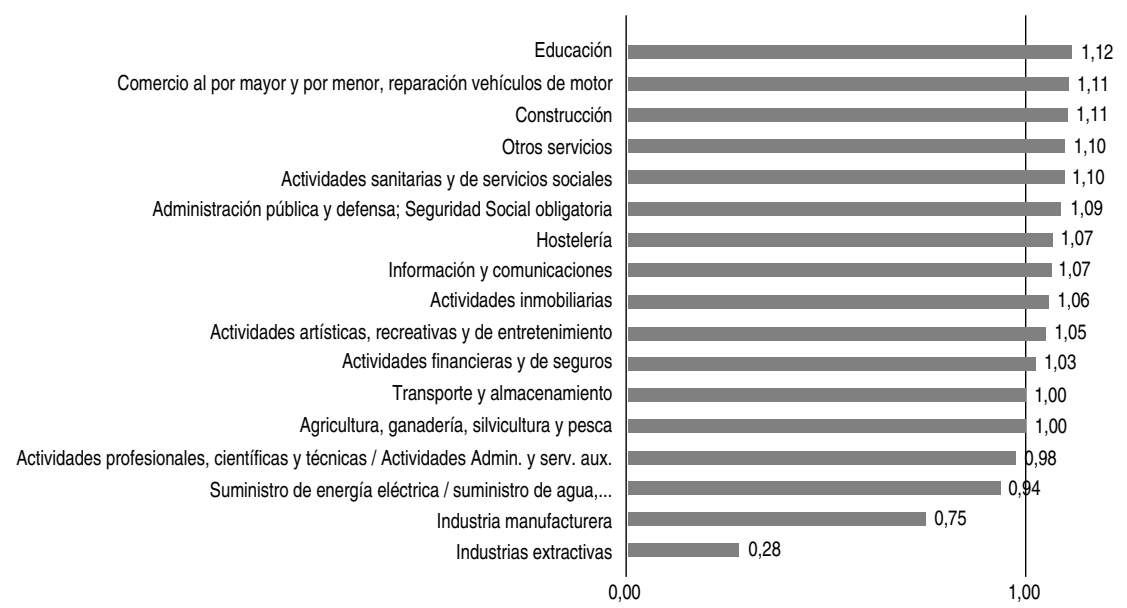

FUENTE: Elaboración propia.

Combinando los valores de los multiplicadores con la distribución sectorial (por número de trabajadores) de las empresas de Economía Social (en este estudio, cooperativas y sociedades laborales), se puede obtener el impacto que una unidad monetaria (en este caso) de aumento de la demanda exógena (por ejemplo, inversión del Sector Público), tendría en la economía si se distribuyera entre las empresas de Economía Social según esta distribución. Realizando el mismo cálculo para el conjunto de la economía y tomando los ratios de la Tabla 1, puede realizarse la comparativa del impacto global en términos de empleo de la inversión en Economía Social frente a la economía global. La Tabla 2 resume estos cálculos 3 .

3.- Los valores sectoriales deben tomarse como un índice de la contribución de dichas ramas y como un indicador de su importancia relativa, puesto que el efecto comparativo específico en cada rama de una inversión unitaria en la misma ya viene reflejada por las ratios de la Tabla 1.

CIRIEC-España, Revista de Economía Pública, Social y Cooperativa

№ 83/2015, pp. 63-81

ISSN: $0213-8093$ 


\section{Tabla 2. Ratio multiplicador de empleo Economía Social / Total. España}

\begin{tabular}{|l|c|}
\hline Agricultura, ganadería, silvicultura y pesca & 2,32 \\
Industrias extractivas & 0,05 \\
Industria manufacturera & 1,27 \\
Suministro de energía eléctrica, gas, vapor y aire acondicionado / Suministro de agua, saneamiento, gestión residuos & 0,06 \\
Construcción & 0,71 \\
Comercio al por mayor y por menor; reparación vehículos motor & 5,27 \\
Transporte y almacenamiento & 0,89 \\
Hostelería & 0,73 \\
Información y comunicaciones & 0,15 \\
Actividades financieras y de seguros & 27,76 \\
Actividades inmobiliarias & 0,20 \\
Actividades profesionales, científicas y técnicas / Actividades administrativas y servicios auxiliares & 0,89 \\
Administración Pública y Defensa; Seguridad Social obligatoria & 0,00 \\
Educación & 5,75 \\
Actividades sanitarias y de servicios sociales & 1,57 \\
Actividades artísticas, recreativas y de entretenimiento & 1,20 \\
Otros servicios & 1,07 \\
Activ. Hogares empleadores personal doméstico, productores bienes y servicios & 0,00 \\
\hline ECONOMíA SOCIAL & 1,91 \\
\hline
\end{tabular}

FUENTE: Elaboración propia.

Los resultados indican que una inyección exógena en las empresas de Economía Social tiene un efecto en el empleo 1,91 veces superior al resultante de realizar dicha inversión en el conjunto de la economía. Esto indica que el impacto de las empresas de Economía Social sobre el empleo en España es especialmente significativo, no sólo por su caracterización cualitativa, sino también por su relevancia cuantitativa. Resultan especialmente significativos los resultados en los sectores de Comercio, Educación y, sobre todo, Actividades financieras y de seguros. La constitución de este tipo de empresas resulta especialmente relevante en estos sectores, cuyas características de producción y operatividad se muestran especialmente adecuadas a las formas jurídicas y de organización interna que son parte de la Economía Social. Si a esto se añade que se trata, de forma similar a otros sectores de servicios en España, de ramas económicas con alta capacidad de multiplicar los impactos y con una importante ratio de empleo (siendo intensivas en trabajo en muchos casos), con una proporción de trabajadores claramente superior en la Economía Social que en el conjunto de la economía, la relevancia de este tipo de forma económica se ve claramente incrementado. 
Por otra parte, hay que indicar que, para comprobar la coherencia de los resultados, se ha repetido el análisis considerando la distribución entre los sectores de actividad de las inyecciones exógenas (demanda exterior, gasto público, etc.) no según el número de trabajadores, sino según el número de empresas. En este caso (ver la tabla A1 en el anexo) los resultados han sido prácticamente los mismos, con un impacto global de la Economía Social en el empleo 1,85 veces superior al de la economía en su conjunto,

\section{5.- Conclusiones}

La importancia de la Economía Social como vía de mantenimiento de unos altos estándares de calidad en el trabajo y su conciliación con el bienestar social, familiar y personal, ya era ampliamente reconocida. Sin embargo, se ha visto incrementada en los últimos años como mecanismo de preservación y creación de empleo en un contexto de crisis como el actual, configurándose como un instrumento, además, de integración laboral de colectivos con mayores problemas laborales. El presente estudio muestra que la inversión en este tipo de empresas puede llevar a efectos en el empleo (tanto de mantenimiento, como de creación, en su caso) muy superiores a la media global, debido, fundamentalmente, a la concentración de este tipo de empresas en sectores determinados y a su propia configuración y definición propias. Así, se cuantifica que inyecciones exógenas en las empresas de Economía Social multiplican la creación de empleo en casi el doble frente al resto de empresas en España.

Teniendo en cuenta, además, que se muestra cómo sectores de servicios cuyas características propias en sus sistemas de organización son especialmente adecuados para asumir las formas jurídicas y de funcionamiento de la Economía Social, resultan especialmente sensibles a shocks exógenos en su demanda, el apoyo 0 asunción de medidas en favor de este tipo de empresas resulta especialmente relevante, no sólo por su caracterización cualitativa, sino también por su relevancia cuantitativa. Esto se ve incrementado por la alta capacidad de multiplicar los impactos y la importante ratio de empleo de estos sectores (básicamente de Servicios), ampliado en los casos recogidos dentro de la Economía Social. Por esto, el uso de este tipo de forma económica como herramienta de política económica debe ser tenido en cuenta por los agentes decisores, especialmente por aquellos que deben velar por la más adecuada asignación de los escasos recursos existentes para la creación o fomento de las empresas. 


\section{6.- Bibliografía}

BARRERA CEREZAL, J.J. (2008): "The Social Economy and public policies", CIRIEC-España, Revista de Economía Pública, Social y Cooperativa, 62, 183-198.

CARDENETE, M.A., FUENTES, P., MAINAR, A. \& RODRÍGUEZ, C. (2015): "Análisis y explotación mediante modelos económicos multisectoriales de la Matriz de Contabilidad Social de Andalucía para 2008", Regional and Sectoral Economic Studies, 15-1, 153-168.

CEPES (2014): ¿Qué es la Economía Social?, http://cepes.es

CHAVES, R. \& MONZÓN, J.L. (Dirs.) (2000): Economía Social y empleo en la Unión Europea, Valencia, ed. CIRIEC-España.

ESCUELA DE ORGANIZACIÓN INDUSTRIAL (EOI) (Varios autores) (2013): El impacto socioeconómico de las entidades de economía social, Fundación EOI, Madrid.

EUROPEAN COMMISSION (2013): Sistema Europeo de Cuentas Nacionales y Regionales de la Unión Europea 2010, Luxembourg, European Commission.

EUROSTAT (2008): Eurostat Manual of Supply, Use and Input-Output Tables, Luxembourg, European Commission.

FERNÁNDEZ, J. \& GONZÁLEZ, P. (2004): "Matrices de Contabilidad Social: una panorámica", Ekonomiaz, 57, 132-163.

FUENTES, P. \& MAINAR, A. (2014): "Growth, Employment and Public Spending in the Social Accounting Matrix of the Spanish Economy for 2008", Actas de la 22nd IIOA Conference, Lisboa. Portugal.

JULIÁ, J. \& SERVER, R.J. (2003): "Social economy companies in the Spanish agricultural sector: delimitation and situation in the context of the European Union", Annals of Public and Cooperative Economics, 74-3, 465-488.

KIRINGAI, J., WANJALA, B., WAIYAKI, N., MUTUNGA, C., NJENGA, G. \& NAFULA, N. (2007): Social Accounting Matrix for Kenya: Methodological Note, Kenya Institute for Public Policy Research and Analysis (KIPPRA).

KUZNETS, S. (1937): Natural income and capital formation, 1919-1935, New York: National Bureau of Economic Research.

LEONTIEF, W. (1941): The structure of American economy, 1919-1929: An empirical application of equilibrium analysis, Cambridge: Harvard University Press. 
MCDOUGALL, R. (1999): "Entropy Theory and RAS are Friends", GTAP Working Papers, 300.

MEYSS (varios años): Anuario de Estadísticas del Ministerio de Empleo y Seguridad Social, (disponible en www.empleo.gob.es/estadisticas).

PYATT, G. \& ROUND, J. (1985): Social Accounting Matrices: a Basis for Planning, The World Bank, Washington.

PYATT, G. \& THORBECKE, E. (1976): Planning Techniques for a Better Future, Ginebra: International Labour Office.

QUESNAY, F. (1758): Tableau Economique (3rd (June 1971) ed.), Augustus M. Kelley Publishers.

RASMUSSEN, P. (1956): Studies in Inter-Sectorial relations, Amsterdam, North-Holland.

REINERT, K. \& ROLAND-HOLST, D. (1997): "Social Accounting Matrices". In: J. Francois, \& K. Reinert, Applied Methods for Trade Policy Analysis: A Handbook, New York: Cambridge University Press.

ROBINSON, S., CATTANEO, A. \& EL-SAID, M. (2001): "Updating and Estimating a Social Accounting Matrix Using Cross Entropy Methods", Economic Systems Research, 13(1), 47-64.

ROUND, J. (2003): "Constructing SAMs for Development Policy Analysis: Lessons Learned and Challenges Ahead", Economic Systems Research, 15, 161-183.

STONE, R. (1947): Measurement of national income and the construction of social accounts, Geneva: United Nations. 


\section{Anexo}

\section{Tabla A1. Ratio multiplicador del número de empresas Economía Social / Total. España}

\begin{tabular}{|l|l|}
\hline Agricultura, ganadería, silvicultura y pesca & 1.68 \\
Industrias extractivas & 0.32 \\
Industria manufacturera & 2.74 \\
Suministro de energía eléctrica, gas, vapor y aire acondicionado / Suministro de agua, saneamiento, gestión residuos & 0.49 \\
Construcción & 1.16 \\
Comercio al por mayor y por menor; reparación vehículos motor & 3.16 \\
Transporte y almacenamiento & 1.08 \\
Hostelería & 0.94 \\
Información y comunicaciones & 0.55 \\
Actividades financieras y de seguros & 5.16 \\
Actividades inmobiliarias & 0.36 \\
Actividades profesionales, científicas y técnicas / Actividades administrativas y servicios auxiliares & 1.30 \\
Administración Pública y Defensa; Seguridad Social obligatoria & 0.01 \\
Educación & 5.57 \\
Actividades sanitarias y de servicios sociales & 1.67 \\
Actividades artísticas, recreativas y de entretenimiento & 1.27 \\
Otros servicios & 0.96 \\
Activ. Hogares empleadores personal doméstico, productores bienes y servicios & 0.01 \\
\hline ECONOMí́ SOCIAL & 1.85 \\
\hline
\end{tabular}

FUENTE: Elaboración propia. 
